\title{
Sleep quality improves during treatment with repetitive transcranial magnetic stimulation (rTMS) in patients with cocaine use disorder: a retrospective observational study
}

Luis J. Gómez Pérez ${ }^{1 \dagger}$, Stefano Cardullo ${ }^{1 \dagger}$, Nicola Cellini ${ }^{2}$, Michela Sarlo ${ }^{2,3}$, Tommaso Monteanni ${ }^{3}$, Antonello Bonci ${ }^{4}$, Alberto Terraneo ${ }^{1}$, Luigi Gallimberti ${ }^{1}$ and Graziella Madeo ${ }^{1 *}$

\begin{abstract}
Background: Sleep disturbance is a prominent and common complaint in people with cocaine use disorder (CUD), either during intake or withdrawal. Repetitive transcranial magnetic stimulation (rTMS) has shown promise as a treatment for CUD. Thus, we evaluated the relationship between self-perceived sleep quality and cocaine use pattern variables in outpatients with CUD undergoing an rTMS protocol targeted at the left dorsolateral prefrontal cortex.

Methods: This is a retrospective observational study including 87 patients diagnosed with CUD according to the DSM-5 criteria. Scores in Pittsburgh Sleep Quality Index (PSQI), Cocaine Craving Questionnaire (CCQ), Beck Depression Inventory-II (BDI-II), Self-rating Anxiety Scale (SAS), and Symptoms checklist 90-Revised (outcome used: Global Severity Index, GSI) were recorded at baseline, and after 5, 30, 60, and 90 days of rTMS treatment. Cocaine use was assessed by self-report and regular urine screens.

Results: Sleep disturbances (PSQI scores $\geq 5$ ) were common in patients at baseline (mean \pm SD; PSQI score baseline: $9.24 \pm 3.89$; PSQI $\geq 5$ in $88.5 \%$ of patients). PSQI scores significantly improved after rTMS treatment (PSQI score Day 90: 6.12 \pm 3.32). Significant and consistent improvements were also seen in craving and in negative-affect symptoms compared to baseline. Considering the lack of a control group, in order to help the conceptualization of the outcomes, we compared the results to a wait-list group $(n=10)$. No significant improvements were observed in the wait-list group in any of the outcome measures.
\end{abstract}

Conclusions: The present findings support the therapeutic role of rTMS interventions for reducing cocaine use and accompanying symptoms such as sleep disturbance and negative-affect symptoms.

Trial registration: ClinicalTrials.gov.NCT03733821.

Keywords: Cocaine use disorder (CUD), Sleep, Craving, Repetitive Transcranial magnetic stimulation (rTMS), Pittsburgh sleep quality index (PSQI), Dorsolateral prefrontal cortex (DLPFC)

\footnotetext{
* Correspondence: graziemadeo@gmail.com

†Luis J. Gómez Pérez and Stefano Cardullo contributed equally to this work.

${ }^{1}$ Novella Fronda Foundation, Piazza Castello, 16 -, 35141 Padua, Italy

Full list of author information is available at the end of the article
}

(c) The Author(s). 2020 Open Access This article is licensed under a Creative Commons Attribution 4.0 International License, which permits use, sharing, adaptation, distribution and reproduction in any medium or format, as long as you give appropriate credit to the original author(s) and the source, provide a link to the Creative Commons licence, and indicate if changes were made. The images or other third party material in this article are included in the article's Creative Commons licence, unless indicated otherwise in a credit line to the material. If material is not included in the article's Creative Commons licence and your intended use is not permitted by statutory regulation or exceeds the permitted use, you will need to obtain permission directly from the copyright holder. To view a copy of this licence, visit http://creativecommons.org/licenses/by/4.0/. The Creative Commons Public Domain Dedication waiver (http://creativecommons.org/publicdomain/zero/1.0/) applies to the data made available in this article, unless otherwise stated in a credit line to the data. 


\section{Background}

Cocaine use disorder (CUD), one of the most prevalent drug use disorders [1], has an estimated $2 \%$ prevalence among young European adults during the past year, and is the third most frequent reason for access to substance abuse treatment programs in Europe [2]. Recovery from CUD is hampered by vulnerability to relapse during the first weeks of abstinence [3]. Several commonly recognized abstinence/withdrawal symptoms such as sleep disturbances, negative affect or craving, may be critical contributors to relapse [3]. In particular, sleep disturbances are often reported by individuals with CUD during either cocaine intake or abstinence [4]. Importantly, poorer self-reported sleep quality has been associated with more frequent use in CUD [5]. Furthermore, in the first weeks of abstinence, polysomnography (PSG) shows worsening sleep, with reductions in duration of rapid eye movement sleep (REM), slow-wave sleep (SWS), and total sleep, and increases in sleep latency [3, 6-9]. These sleep abnormalities are long-lasting [6] and related to the severity of withdrawal symptoms and clinical outcomes [10]. However, the causal relationship between sleep disturbances and CUD still needs to be clarified [11]. Interestingly, sleep deprivation and psychostimulants share similar neurobiological effects on several neurotransmitter pathways, including the dopaminergic system [12-16]. Hence, the development of interventions aiming to rewire the affected brain circuitry might lead to clinical improvements of addictive behaviors and better regulation of the sleep patterns in CUD patients.

Recently, CUD and other addictive disorders have been treated with transcranial magnetic stimulation (TMS), a well-known and safe neuromodulatory technique, which consists in inducing electrical activity in the human brain through the application of magnetic pulses $[17,18]$. Compelling evidence has demonstrated that repetitive TMS (rTMS) has short- and long-term effects on neural activity, both locally, under the stimulating coil, and at the brain-network level [19]. Furthermore, rTMS may have a positive longterm impact on behaviors related to craving, drug intake, and relapse. Indeed, clinical pilot studies on CUD, albeit preliminary, are now supporting the potential role of rTMS in decreasing cocaine craving [20-23] and intake [20, 23, 24]. Polysomnographic studies in healthy volunteers have shown that rTMS induces opposite changes in sleep architecture to those found in people with CUD, such as a prolongation of REM latency [25] or a marked increase in slow-wave sleep [26]. TMS may also have therapeutic effects on insomnia, restless-leg syndrome, and sleep disturbances associated with epilepsy, depression, and post-traumatic stress disorder [27]. In particular, rTMS stimulation of the dorsolateral prefrontal cortex (DLPFC) has shown benefits in patients with chronic primary insomnia, by reducing sleep latency and increasing total sleep time and REM latency $[27,28]$.
Together, these findings support the hypothesis that rTMS could modulate sleep architecture in ways that might benefit people with CUD. To our knowledge, so far only two studies attempted to investigate the effect of a rTMS treatment on sleep disturbances in substance use disorders. One study assessed the sleep quality in abstinent inpatients dependent on heroin or methamphetamine [29] using the Pittsburgh Sleep Quality Index (PSQI). The authors found that five sessions of rTMS stimulation over the primary motor cortex (M1) administered for six consecutive weeks significantly improved the sleep quality of substance dependent inpatients in early abstinence compared to both sham and control wait-list groups. Another study evaluated the sleep disturbances in CUD patients, who underwent a high frequency rTMS protocol over the left-DLPFC, by using the Insomnia Severity Index (ISI) [30]. They found a global improvement of the ISI score that, however, did not reach the statistical significance. The conflicting nature of these findings is dependent on several factors, including sample size, stimulation protocol parameters, open label-design, outcome measure tools.

In the present study, we hypothesized that rTMS treatment would be accompanied by an improvement in sleep disturbances reported by CUD patients during the abstinence/withdrawal period. Therefore, we investigated the modulation of sleep quality reported by CUD patients undergoing an rTMS stimulation protocol applied to the left DLPFC [20], before treatment (baseline) and during the treatment at 5, 30, 60, and 90 days. We also assessed accompanying withdrawal symptoms, such as craving, depression, and anxiety, during the whole period of treatment.

\section{Methods}

\section{Participant selection}

This current study is a retrospective chart review of data from 87 patients with CUD who were treated with an rTMS protocol from 2015 to 2017 in an open label, no sham control study. On the day of clinical intake, patients provided a written informed consent authorizing the use of their data for research. Patients were informed that the data collected would be managed according to the law on privacy and the Legislative Decree $\mathrm{N}^{\circ} .196$ of June 30, 2003, "Personal Data Protection Code", warranting anonymity. The approval for the protocol, limited to the retrospective chart review, was obtained from the Ethical Committee for the Psychological Research of the School of Psychology, University of Padova (protocol number: 2551).

The current retrospective analysis is listed at www. clinicaltrials.gov (Registration Number: NCT03733821). Participants were recruited after referral to a specialty outpatient clinic, Center for Addiction in Padua (Italy). 
Participants were 22 to 57 years old and met diagnostic criteria for CUD according to the Diagnostic and Statistical Manual of Mental Disorders - 5 (DSM 5), as assessed by a clinical psychiatrist specialized in substance use disorders (SUDs) [31]. Each patient was followed by the same clinician. Data were entered by the clinical staff into the electronic medical records of the outpatient clinic. Considering the study's design and the lack of a control group, to help conceptualize the results, we compared the outcomes of the recruited sample to a wait-list group. Ten participants, with comparable clinic characteristics, were assessed 30 days before the beginning of treatment and at Day 0. A 30-day period is the maximum time allowed for the waiting time in our clinical setting considering the severity of the disease.

Exclusion criteria included a prior history of other psychiatric diseases, including major depression, schizophrenia, bipolar disorder or other psychosis, current alcohol, and other substance abuse or dependence (excluding cocaine, nicotine, and caffeine), pregnancy or breastfeeding, personality disorders or sleep disturbances deemed to be the primary disease, current unstable medical illness, substantial neurological illness, and any contraindication for rTMS (including implanted metal and devices in the body, or history of epilepsy). Participants were required to keep medication use stable throughout the study. During the whole period of observation, cocaine use was assessed either via a urine drug test, at each visit, or via reports from the patient or significant others. The urine drug screen panel also included the following: morphine, methadone, THC, phencyclidine, amphetamine, and methamphetamine.

\section{Outcome measures}

The primary outcome measure, the perceived sleep quality, was assessed by the Pittsburgh Sleep Quality Index (PSQI) [32], commonly used in clinical and research settings [33]. Secondary outcome measures were craving, depression, anxiety, and other negative affect symptoms, assessed with the following scales: Cocaine Craving Questionnaire (CCQ) [34], Beck Depression Inventory II (BDI - II) [35], Self-rating Anxiety Scale (SAS) [36], and Symptoms checklist 90 - Revised (SCL-90-R) [37]. Participants were assessed at baseline, immediately after completion of the first week of treatment (Day 5), and 30,60 , and 90 days after the beginning of treatment (Day 30 - Day 60 - Day 90). The instructions of BDI-II require the participant to consider the last 2 weeks preceding the test; thus, it was not included in the assessment at Day 5. Several participants did not complete every scale at every time point, for the main following reasons: clinical response, missing follow-up visit, missing TMS session, and refusal. We included all participants who completed outcome measures for at least two time points, including the baseline. Therefore, of the $142 \mathrm{pa}$ tients initially assessed for eligibility, 55 were excluded for the lack of outcomes measure other than baseline. A flow diagram describing the progress of the patients throughout the study is shown in Fig. 1.

\section{rTMS treatment}

The rTMS stimulation protocol was administered by a trained clinical physiologist. All patients were seated in a comfortable chair while TMS stimuli were delivered to the left DLPFC (MNI coordinates: $\mathrm{x}=-50, \mathrm{y}=30, \mathrm{z}=$ 36). The stimulator device was a MagPro R30 with a Cool-B80 butterfly coil (MagVenture, Farum, Denmark). The target site of stimulation was identified by using an optical TMS Navigator (LOCALITE, St. Augustin, Germany) and a magnetic resonance image (MRI) template. Resting motor threshold (rMT) was evaluated as previously described by Terraneo [20]. The stimulation protocol parameters were: $15 \mathrm{~Hz}$ frequency, $100 \%$ of rMT, 40 trains, 60 pulses per train, $15 \mathrm{~s}$ intertrain interval, and 2400 pulses per session. Patients received 2 sessions per day for the first consecutive 5 days of treatment (10 sessions), and 2 sessions per week for the next 12 weeks. The time interval between the two sessions each day was 45-60 min. The number of rTMS sessions was adjusted according to the specific needs of each patient and the clinical response during the 90 days of treatment. At each session, adverse events were also selfreported. Each patient received multidisciplinary support tailored to his or her needs.

\section{Statistical analysis}

Two independent paired-sample $t$ tests were performed to compare the number of days of cocaine use during the 30 days before the beginning of treatment and the use in the first 30 days and last 30 days of treatment. One-way ANOVAs using the time-point as a 5 levels independent variable ("Baseline", "Day 5", "Day 30", "Day 60", "Day 90") were computed for each dependent variable (PSQI, CCQ, BDI-II, SAS, GSI) in order to estimate the overall effect of treatment. For pairwise comparisons between timepoints, we used Tukey tests. Thereafter, for examining the best predictor of change and because not all participants provided complete data, we used multilevel models, which, unlike repeated-measures analysis of variance (ANOVA), do not require listwise deletion for missing data. By assuming a different "baseline" for each subject, we controlled the innate between-subject differences. For each dependent variable, we initially tested the following predictors: number of days since the beginning of rTMS treatment, years of education, age at the beginning of rTMS treatment, age at the first experience with cocaine, and age at the time of addiction to cocaine. As time-varying covariates, we included the 


\section{CONSORT 2010 Flow Diagram}

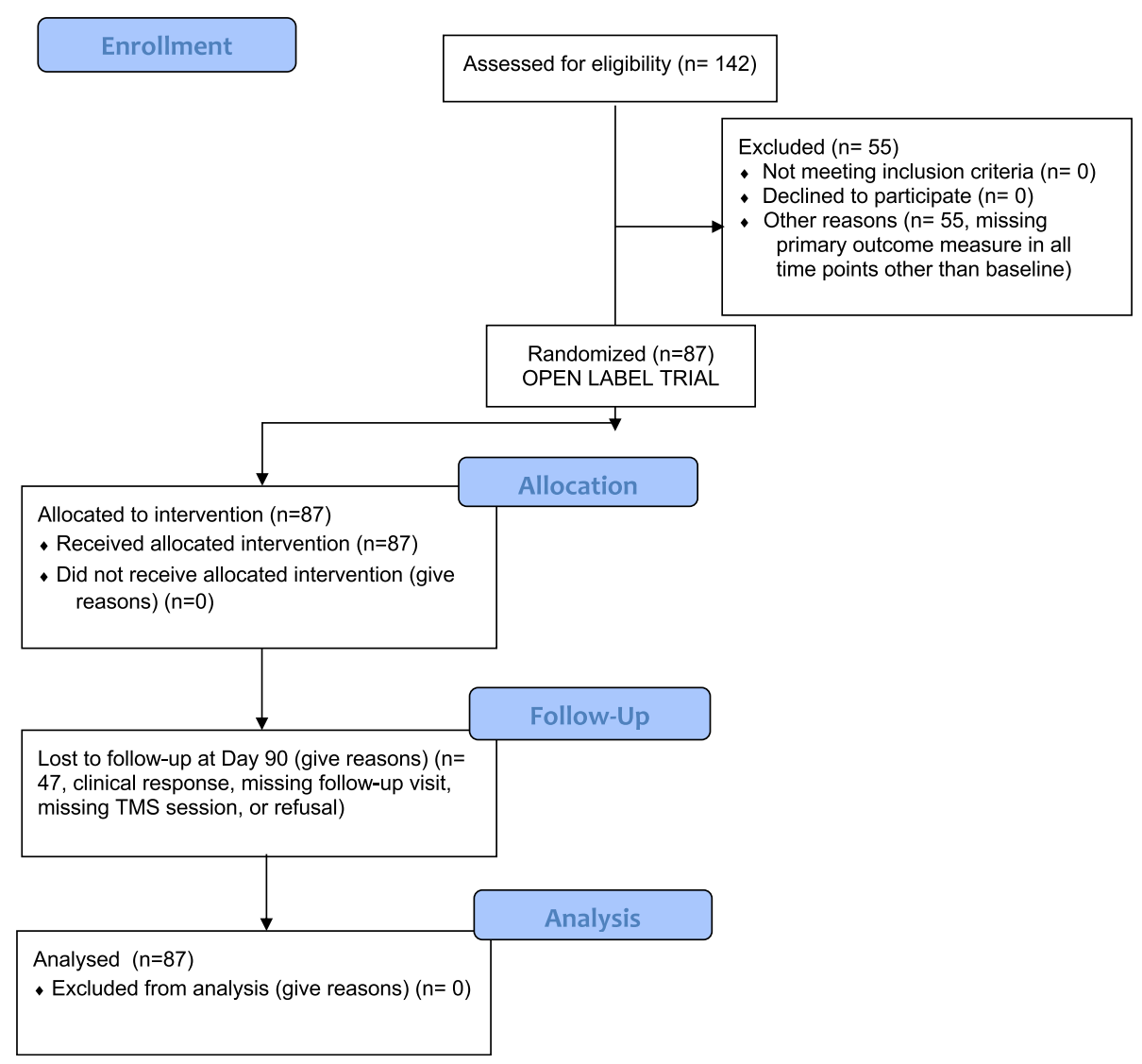

Fig. 1 Consort 2010 Flow Diagram of the study

number of rTMS sessions in the preceding 30 days and cocaine use, as defined by the number of positive urine specimens or use reported to the clinician in the preceding 30 days. We used a random intercept for each participant. We did not test for sex differences because most participants were male. For each model, we reported the estimated fit by maximum likelihood and the t-tests with Satterthwaite degrees of freedom.

Lastly, considering the lack of a control group and trying to give a better context to the results, we compared the outcomes to a wait-list group $(n=10)$. A small sample of equal numerosity and controlled for PSQI severity at baseline, years of education, age at the beginning of rTMS treatment, age at the first experience with cocaine, and age at the time of addiction to cocaine, was randomly selected from the 87 patients recruited for the study. Patients of the wait-list group were assessed 30 days before the beginning of treatment and at Day 0. A comparable 30-days-window of observation was chosen from the active sub-sample randomly selected (Day 0 Day 30). ANOVAs for each dependent variable with the interaction Group ("active" Vs "wait-list") * Time-point ("Pre" Vs "Post") was run. For pairwise comparisons between timepoints and groups, we used Tukey tests.

Data were expressed as mean \pm standard deviation (SD), unless otherwise specified; alpha was set at $<0.05$, two-tailed. All the analyses were performed using RStudio versions 1.1.453 [38] with $\mathrm{R}$ version 3.5.0 [39] and the packages lme4 [40], lmerTest [41], and effects [42].

\section{Results}

\section{Demographic and clinical characteristics and treatment} parameters

Full demographic and clinical characteristics of the participants are presented in Table 1. The total sample consisted of 87 patients, 2 females and 85 males, aged between 22 and $57(37.67 \pm 7.53)$. Treatment variables are specified in Table 1. After the first week of treatment, the number of rTMS sessions was not uniform across patients, because it was adjusted based on clinical response and because several participants missed appointments. Between Day 5 and Day 30, one patient out 
Table 1 Demographic and clinical characteristics of the participants

\begin{tabular}{ll}
\hline Variables & All $(\boldsymbol{n}=87)$ \\
\hline Age (years) & $37.67(7.53)$ \\
Gender (female/male) & $2 / 85$ \\
Education (years) & $12.51(3.2)$ \\
Age at first experience (years) & $20.55(5.65)$ \\
Age at addiction (years) & $28.62(8.8)$ \\
Cocaine use 30 days before baseline (n. of days; \%) & $19.17(11.45)$ \\
Daily & $47 \%$ \\
Weekly & $33 \%$ \\
Monthly & $20 \%$ \\
rTMS sessions number & $29.17(6.34)$ \\
PSQI score $\geq 5$ at baseline [\%] & 88.5 \\
CCQ score at baseline & $12.66(10.93)$ \\
BDI-Il score at baseline & $18.98(9.91)$ \\
SAS score at baseline & $47.93(10.01)$ \\
GSI score at baseline & $65.91(16.53)$
\end{tabular}

Data are presented as mean (standard deviation), unless otherwise specified. $B D I-I I$ Beck Depression Inventory-II; CCQ Cocaine Craving Questionnaire; GSI Global Severity Index of the Symptoms checklist 90 - Revised; PSQI Pittsburgh Sleep Quality Index; rTMS repetitive Transcranial Magnetic Stimulation; SAS Self-rating Anxiety Scale

of $87(1.15 \%)$ did not receive any rTMS treatment; the other 86 patients (98.85\%) received 2-22 sessions $(6.91 \pm 2.68)$. Between Day 30 and Day 60, 82 patients $(94.25 \%)$ received $1-12$ sessions $(7.47 \pm 2.55)$. Between Day 60 and Day 90, 61 patients $(70.11 \%)$ received $2-17$ sessions $(5.55 \pm 3.09)$.

\section{Cocaine use}

At the beginning of treatment $47 \%$ of patient reported a daily use of cocaine, $33 \%$ used weakly, and $20 \%$ monthly. The mean number of cocaine uses per patient was of
19.17 days $(\mathrm{SD} \pm 11.45)$ that significantly decreased at Day $30(0.51 \pm 0.95 ; \mathrm{t}(81)=14.56, p<.001)$ and was stable at Day $90(0.84 \pm 1.5 ; \mathrm{t}(64)=12.66, p<.001)$. $71.9 \%$ of patients were abstinent during the first 30 days of treatment and $66.1 \%$ were abstinent at the end of treatment. Conversely, patients from the wait-list group did not show any significant changes of the cocaine use during the 30-day waiting period ( $\mathrm{t}(9)=0.68, p=.50$ ).

\section{Primary outcome: changes in subjective quality and pattern of sleep}

The first goal of our analyses was to investigate changes of subjective quality and pattern of sleep in outpatients undergoing rTMS of left DLPFC for CUD. Changes of PSQI score during the observation period are summarized in Table 2. At baseline, most patients $88.5 \%$ (77 out of 87) had a PSQI score greater than or equal to 5, indicated sleep disturbance. PSQI scores significantly improved at each timepoint after the first week of rTMS treatment $(\mathrm{F}(4,303)=20.81, p<0.001)$. Pairwise comparisons showed that PSQI scores at Day 5 were significantly lower than those at baseline (Day 5: $5.09 \pm 3.33$ ); Baseline: $9.24 \pm 3.89 ; p<0.001)$. This improvement was maintained through the three subsequence time points: Day $30(5 \pm 3.13 ; p<0.001)$, Day $60(5.28 \pm 3.47 ; p<$ 0.001 ), and Day 90 (6.12 $\pm 3.32 ; p<0.001$, Fig. 2).

In a separate model, we showed that improvements in PSQI score were significantly related to the number of rTMS sessions in the preceding 30 days $(t$ (196.33) = 3.31, $p=0.001$ ), with greater number of rTMS sessions associated with a lower PSQI score (i.e., higher global sleep quality) (Fig. 3). This model controlled for number of cocaine use over the same time period (associated with worse sleep: $\mathrm{t}(225.4)=4.94, p<0.001)$, with greater use of cocaine associated with a higher PSQI score (i.e., lower global sleep quality), and for age at the first

Table 2 Clinic outcome scores: change from baseline during rTMS treatment for overall study population

\begin{tabular}{|c|c|c|c|c|c|}
\hline & Baseline & Day 5 & Day 30 & Day 60 & Day 90 \\
\hline$\overline{\text { PSQI }^{\mathrm{a}}}$ & $9.24(3.89)$ & $5.09(3.33)$ & $5(3.13)$ & $5.28(3.47)$ & $6.12(3.32)$ \\
\hline Change from Baseline & & $-4.15(0.53) *$ & $-4.24(0.58) *$ & $-3.96(0.67) *$ & $-3.12(0.66) *$ \\
\hline $\mathrm{CCQ}^{\mathrm{b}}$ & $12.67(10.93)$ & $2.21(3.29)$ & $1.34(2.79)$ & $1.84(4)$ & $3.8(6.5)$ \\
\hline Change from Baseline & & $-10.45(1.15) *$ & $-11.32(1.20) *$ & $-10.81(1.41) *$ & $-8.86(1.38) *$ \\
\hline BDI-II ${ }^{c}$ & $18.99(9.91)$ & $\_\S$ & $5.09(6.45)$ & $5.33(7.67)$ & $6.72(7.2)$ \\
\hline Change from Baseline & & & $-13.89(1.38) *$ & $-13.65(1.64) *$ & $-12.26(1.58) *$ \\
\hline SAS $^{d}$ & $47.93(10.01)$ & $36.11(8.45)$ & $35.97(9.44)$ & $35.33(9.52)$ & 38.09 (7.38) \\
\hline Change from Baseline & & $-11.81(2.04) *$ & $-11.96(1.54) *$ & $-12.60(1.87) *$ & $-9.83(1.77) *$ \\
\hline $\mathrm{GSI}^{\mathrm{e}}$ & $65.91(16.53)$ & $46.69(12.17)$ & $47.67(14.46)$ & $44.49(10.92)$ & $46.46(9.56)$ \\
\hline Change from Baseline & & $-19.22(2.08) *$ & $-18.24(2.25) *$ & $-21.42(2.67) *$ & $-19.45(2.59) *$ \\
\hline
\end{tabular}

Data are presented as Mean (SD);

${ }^{*} p$ value $<.001 ;{ }^{\S} \mathrm{BDI}-$ Il was not administered at day 5 because it refers to the last 2 weeks;

${ }^{a}$ Pittsburgh Sleep Quality Inventory - general sleep quality index; ${ }^{b}$ Cocaine Craving Questionnaire; ${ }^{c}$ Beck Depression Inventory - II; ${ }^{d}$ Self-rating Anxiety Scale;

e Global Severity Index from Symptoms Checklist - 90 - Revised 

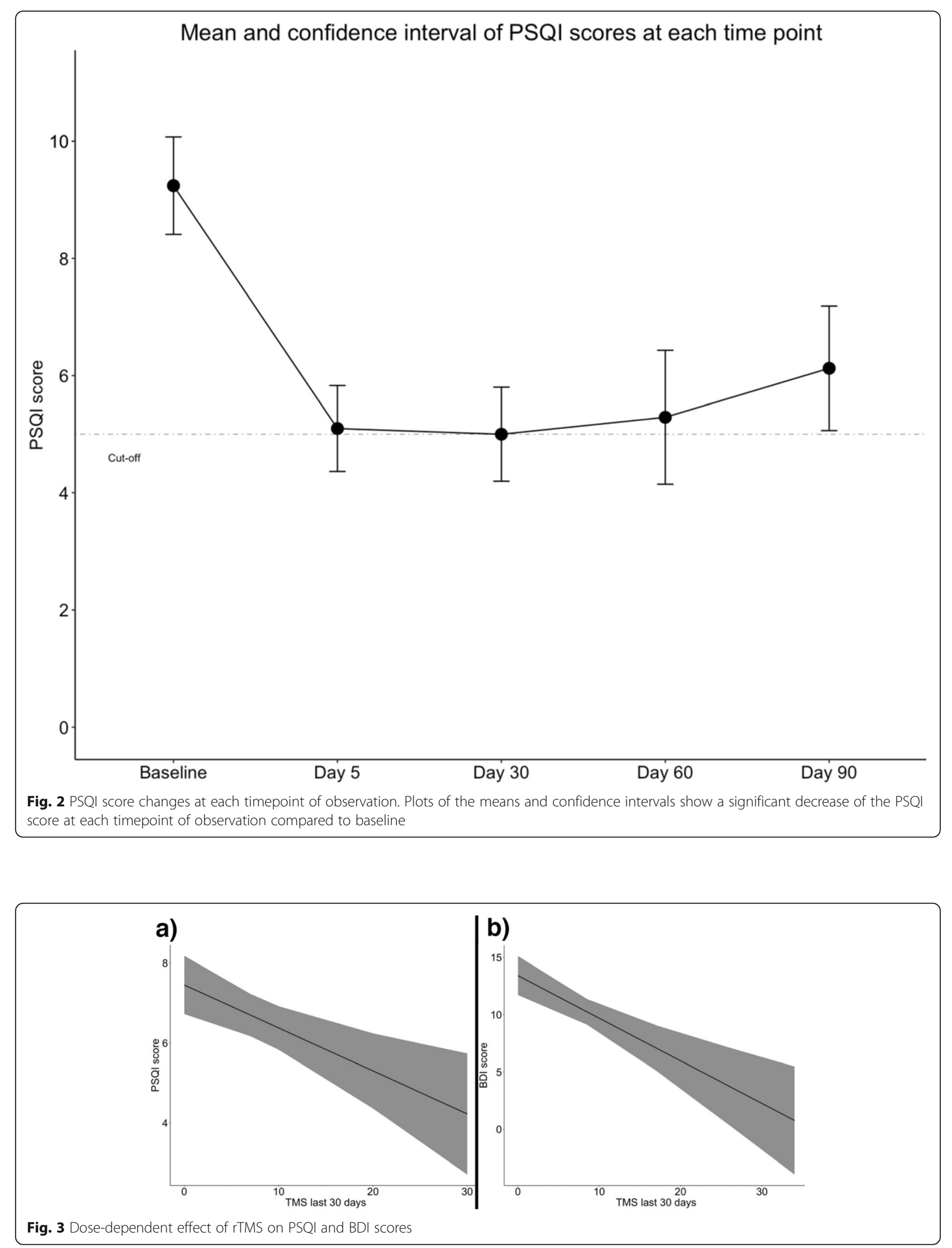
experience with cocaine ( $\mathrm{t}(82)=-1.9, p=0.051)$, suggesting that CUD subjects with an earlier cocaine experience tend to show the greater sleep improvement. From the $t$ of -3.31 and the degrees of freedom [43], we can calculate a model-adjusted $r_{\text {effect }}$ size between session frequency and subsequent sleep disturbance: $r=-.23$ (95\% CL -0.42 to -0.02$)$. In this model, the main effect of Time was not significant $(\mathrm{t}(197.35)=-0.876, p=.38$; $\mathrm{r}_{\text {effect }}=-.06(-.15$ to .27$)$, suggesting that time in treatment had little benefit over and above that of rTMS dosage and cocaine abstinence.

\section{Secondary outcomes: craving, depression, and anxiety}

Like sleep disturbances, these affective symptoms significantly decreased over time, as reflected in: CCQ (F (4, $255)=32.27, p<0.001)$, BDI-II $\quad(\mathrm{F} \quad(3,219)=46.34, p<$ $0.001)$, GSI $(\mathrm{F}(4,306)=32, p<0.001)$, and SAS (F $(4$, $245)=22.51, p<0.001)$. All the secondary outcome measures showed a significant improvement at Day 5 of treatment (CCQ: $2.21 \pm 3.29, p<0.001$; GSI: $46.69 \pm$ $12.17, p<0.001$; SAS: $36.11 \pm 8.45, p<0.001)$. This improvement was stable across the subsequent three time points: Day 30 (CCQ: $1.34 \pm 2.79, p<0.001$; BDI-II: $5.09 \pm 6.45, p<0.001$; GSI: $47.66 \pm 14.46, p<0.001$; SAS: $35.96 \pm 9.44, p<0.001$ ), Day 60 (CCQ: $1.84 \pm 4, p<0.001$; BDI-II: $5.33 \pm 7.67, p<0.001$; GSI: $44.49 \pm 10.92, p<$ 0.001 ; SAS: $35.33 \pm 9.52, p<0.001$ ), and Day 90 (CCQ: $3.8 \pm 6.5, p<0.001$; BDI-II: $6.72 \pm 7.2, p<0.001$; GSI: $46.46 \pm 9.56, p<0.001$; SAS: $38.09 \pm 7.38, p<0.001)$.

In separate models, we found that these improvements were associated with both the number of rTMS sessions and the use of cocaine in the preceding 30 days. The number of rTMS sessions was associated with the degree of reduction in craving as assessed by CCQ (t (162.09) = $-2.99, p=0.003)$, in a model that controlled for frequency of cocaine use during the same period $(\mathrm{t}$ $(200.86)=6.01, p<0.001)$. From the $\mathrm{t}$ of -2.99 and the degrees of freedom, we can calculate a model-adjusted $r_{\text {effect }}$ size between session frequency and subsequent cocaine craving: $\mathrm{r}=-.23$ (95\% CL -0.42 to -0.02$)$. In the model for CCQ, the main effect of Time was not significant $\left(\mathrm{t}(172.96)=-1.341, p=.18 ; \mathrm{r}_{\text {effect }}=-.10(-.30\right.$ to .11), again suggesting that time in treatment had little benefit over and above that of rTMS dosage and cocaine abstinence.

Similar associations occurred for reductions in BDI-II scores (number of rTMS sessions, $\mathrm{t}(183.68)=-4.255$, $p<0.001$; use of cocaine, $\mathrm{t}(207.82)=2.789, p=0.005)$ (Fig. 3), GSI scores (number of rTMS sessions, t $(193.01)=-3.087, p=0.002$; use of cocaine, $\mathrm{t}(220.54)=$ 3.143, $p=0.001$ ), and SAS anxiety scores (number of rTMS sessions, $\mathrm{t}(165.11)=-4.026, p<0.001$; use of cocaine, $\mathrm{t}(195.19)=2.637, p=0.009)$. Model-adjusted $\mathrm{r}_{\text {effect }}$ sizes for rTMS dose effects on subsequent BDI, GSI and SAS were, respectively, 0.30 ( -0.48 to -0.10$), 0.22$ (0.41 to -0.01$)$, and $0.30(-0.48$ to -0.10$)$. In each of these models, the main effect of Time was significant ( $\mathrm{r}_{\text {effect }}$ sizes 0.27 to 0.33 ), suggesting that, for depression and anxiety, time in treatment did confer some generalized benefit over and above that of rTMS dosage and cocaine abstinence (Table 3).

\section{Wait-list comparisons}

Changes of outcome scores during the 30-days observation period are summarized in Table 4 . With regard to PSQI, results of ANOVA showed a significant main effect of Time $(F(1,36)=5.43, p=0.02)$ and of the interaction Time*Group (F $(1,36)=7.73, p=0.008$; Fig. 4). Pairwise comparison showed no differences between active and wait-list group at first assessment ( $\mathrm{t}(36)=$ $1.649, p=0.10)$, whilst become significant 30 days later ( $\mathrm{t}$ $(36)=-2.283, p=0.02)$. Patients in the active group significantly improved overtime ( $\mathrm{t}(36)=3.615, p<0.001$ ), while patients in the wait-list remained stable $(\mathrm{t}(36)=-$ $0.317, p=0.75$ ). Similarly, CCQ scores remained stable in the wait-list patients $(\mathrm{t}(36)=0.611, p=0.54)$ and significantly improved in the active group ( $\mathrm{t}(36)=3.748$, $p<0.001)$. There was no statistically significant

Table 3 Beta values of the significant effects in each model

\begin{tabular}{|c|c|c|c|c|c|}
\hline Predictors & $\mathrm{PSQI}^{\mathrm{a}}$ & $\mathrm{CCQ}^{\mathbf{b}}$ & $\left.\mathrm{BD}\right|^{\mathrm{c}}$ & $S A S^{\mathbf{d}}$ & $\mathrm{GSI}^{\mathbf{e}}$ \\
\hline TMS last 30 days & $-0.11(0.03) *$ & $-0.22(0.07) *$ & $-0.37(0.08)^{* *}$ & $-0.34(0.08) * *$ & $-0.40(0.13) *$ \\
\hline Use last 30 days & $0.13(0.02) * *$ & $0.37(0.06) * *$ & $0.20(0.07) *$ & $0.18(0.07) *$ & $0.33(0.10) *$ \\
\hline Time & & & $-0.09(0.02)^{* *}$ & $-0.07(0.01)^{* *}$ & $-0.13(0.02)^{* *}$ \\
\hline First experience & & & & $-0.50(0.17) *$ & \\
\hline \multicolumn{6}{|l|}{ Addiction age } \\
\hline \multicolumn{6}{|l|}{ Age } \\
\hline Education & & & & & \\
\hline
\end{tabular}

Data are presented as estimate (Standard Error)

${ }^{*} p<0.01$; ${ }^{* *} p<0.001$;

${ }^{a}$ Pittsburgh Sleep Quality Inventory - general sleep quality index; ${ }^{b}$ Cocaine Craving Questionnaire;

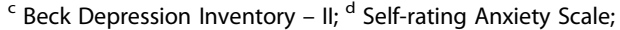

e Global Severity Index from Symptoms Checklist - 90 - Revised 
Table 4 Changes of outcome scores during the 30-days observation period in active and in wait-list group

\begin{tabular}{|c|c|c|c|c|}
\hline & Active group & & Wait-list gro & \\
\hline & Day 0 & Day 30 & Day - 30 & Day 0 \\
\hline PSQI $^{a}$ & $9.00(4.85)$ & $3.3(1.56)$ & $6.4(3.33)$ & $6.9(3.54)$ \\
\hline Change from first assessment & & $-5.7(1.57) *$ & & $0.5(1.57)$ \\
\hline $\mathrm{CCQ}^{\mathrm{b}}$ & $18.8(9.25)$ & $1.00(2.82)$ & $24.8(13.79)$ & $21.9(12.93)$ \\
\hline Change from first assessment & & $-17.8(4.74) *$ & & $-2.9(4.74)$ \\
\hline BDI-II & $18.7(8.17)$ & $2.7(2.31)$ & $15.6(7.48)$ & $14.1(7.35)$ \\
\hline Change from first assessment & & $-16(3.01) *$ & & $-1.5(3.01)$ \\
\hline SAS $^{d}$ & $47.62(9.04)$ & $32.62(6.54)$ & $45.12(8.21)$ & $43.00(8.94)$ \\
\hline Change from first assessment & & $-15(3.68) *$ & & $-2.12(3.68)$ \\
\hline $\mathrm{GSI}^{\mathrm{e}}$ & $68.13(17.90)$ & $42.08(7.31)$ & $61.95(9.70)$ & $57.85(11.74)$ \\
\hline Change from first assessment & & $-26.05(5.50) *$ & & $-4.1(5.50)$ \\
\hline
\end{tabular}

Data are presented as Mean (SD);

* $p$ value <.001; § A small sample of equal numerosity and clinical characteristics of wait-list randomly selected from the 87 patients recruited for the study

${ }^{a}$ Pittsburgh Sleep Quality Inventory - general sleep quality index; ${ }^{b}$ Cocaine Craving Questionnaire; ${ }^{c}$ Beck Depression Inventory - II; ${ }^{d}$ Self-rating Anxiety Scale;

e Global Severity Index from Symptoms Checklist - 90 - Revised

difference in craving scores between groups at first assessment ( $\mathrm{t}(36)=-1.263, p=0.21$ ). At the end of 30 days considered, the difference between groups was significant ( $\mathrm{t}(36)=-4.401, p<0.001$ ). Same pattern was observed for the other secondary outcomes. Wait-list patients remained stable overtime (BDI-II: $\mathrm{t}(36)=0.497$, $p=0.62$; SAS: $\mathrm{t}(36)=0.576, p=0.56$; GSI: $\mathrm{t}(36)=0.746$, $p=0.46)$, compared to the improvement observed in the active patients (BDI-II: $\mathrm{t}(36)=5.298, p<0.001$; SAS: $\mathrm{t}$ $(36)=4.067, p<0.001$; GSI: $\mathrm{t}(36)=4.731, p<0.001)$.

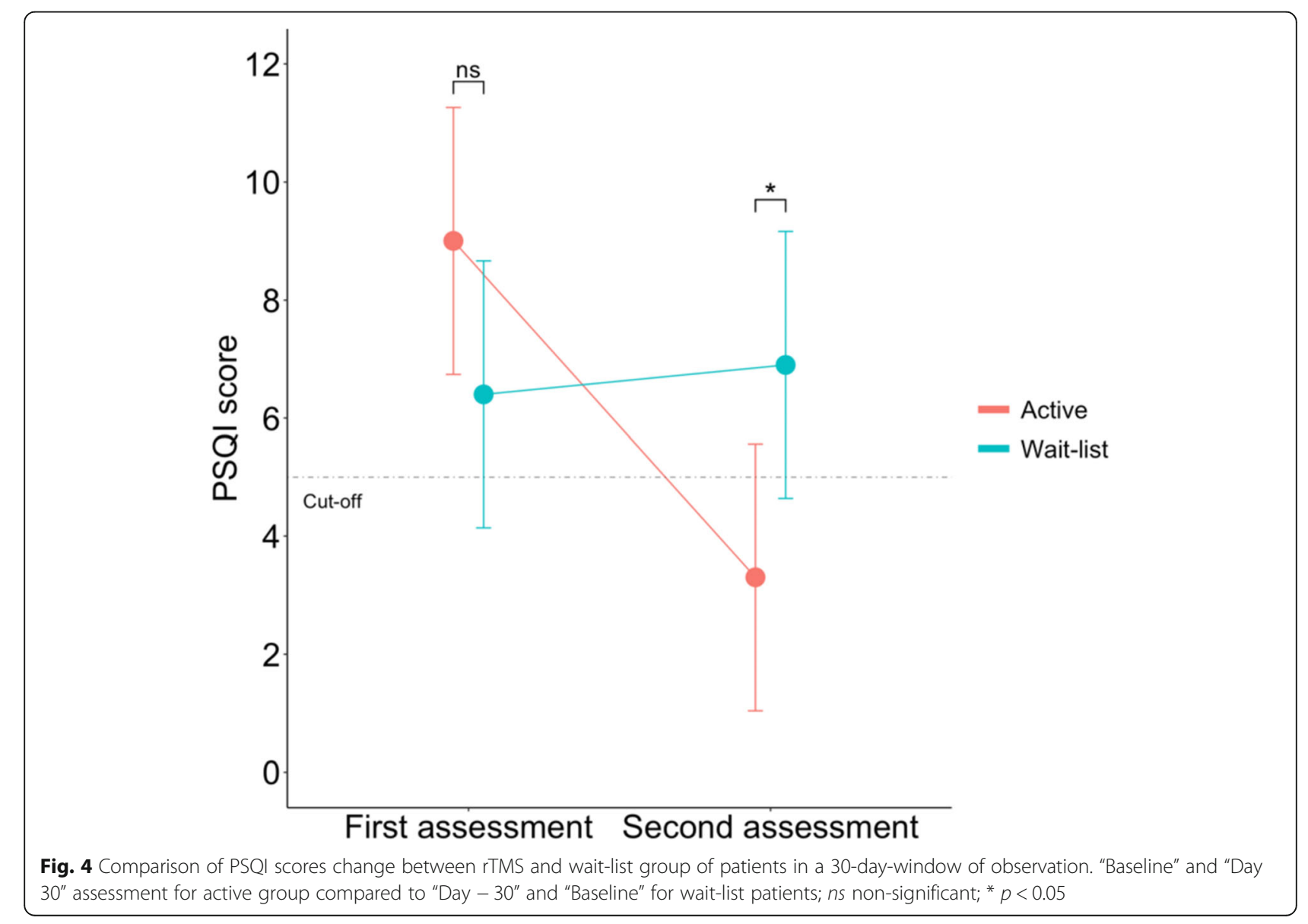




\section{Safety}

None of these 87 patients reported any serious adverse events during the course of the study. There were no seizures, syncopes, neurological complications, or subjective complaints about memory or concentration impairment limiting the treatment and no patient discontinued treatment prematurely due to intolerable stimulation, pain, or other adverse effects such as headache, vertigo, or fatigue.

\section{Discussion}

The main aim of this data analysis was to evaluate changes in commonly experienced symptoms of cocaine abstinence, including sleep disturbances, depression, craving, and anxiety [44], in a large cohort of patients with CUD undergoing rTMS of the left DLPFC. In addition, we examined for the first time whether the "dose" (number of sessions) of rTMS would predict subsequent sleep quality, anxiety, and depressive symptoms in people with CUD, over and above their frequency of cocaine use.

At the beginning of treatment 41 (47.1\%) of our 87 patients reported a daily use of cocaine. We observed a significant decrease of frequency of cocaine use in the first 30 days of treatment, which was maintained at the end of our 90-day rTMS treatment period. No change was observed in the wait-list group. With regard to the perceived quality of sleep, at baseline, 77 $(88.5 \%)$ of our 87 treatment-seeking CUD patients had PSQI scores indicating a poor quality of sleep [32]. This is in agreement with previous findings of sleep disturbances associated with either cocaine dependence or the early withdrawal/abstinence period $[45,46]$. At the end of the first week of rTMS treatment (sessions twice a day, for 5 consecutive days), PSQI scores improved. This improvement remained stable throughout the 90-day rTMS treatment period. Importantly, at Day 90 only $62.5 \%$ of the patients reported a PSQI score equal or higher than 5 .

Sleep disturbances in CUD patients are often treated empirically with pharmacological agents, including sedative, antidepressant, or antiepileptic drugs, with no proven long-term efficacy. Moreover, the pharmacological approach does not affect the pattern of substance use [45]. Only Modafinil (an FDA-approved medication for sleepiness associated with narcolepsy, and shift work sleep disorder) has been shown to improve sleep disruption in CUD patients [47]. However, results of its efficacy on the cocaine use pattern are discordant, as some studies reported an improvement of cocaine use and craving [47-49] not confirmed by others [50-53]. Furthermore, the occurrence of adverse events are often accompanied with dropouts [48-50, 53], which, instead, were not observed in the present study.
To our knowledge, this is the first study investigating changes in sleep quality in patients with CUD undergoing rTMS. Indeed, few published studies have examined changes of sleep quality during or after rTMS treatment, and the results have been mixed. In a randomized clinical trial, people with methamphetamine use disorder undergoing high-frequency rTMS of left DLPFC showed significant improvements in withdrawal symptoms, including self-perceived sleep difficulties, compared to a sham control group [54]; however, no such improvement in sleep disturbance was seen in a similar sham-controlled trial [55]. The conflicting results could reflect differences in sample size, duration of follow-up period, number of rTMS sessions, and intensity of stimulation [56].

Compelling evidence already supports a beneficial effect of rTMS for primary sleep disorders [28], and for sleep disturbances comorbid with other neuropsychiatric disorders, such as obsessive and compulsive disorder [57], focal epilepsy [58], depression [59], and heroin and methamphetamine addiction [29]. Both high and lowfrequency rTMS can modulate total sleep and total wake times, sleep latency, sleep efficiency, and other aspects of sleep architecture [28, 59, 60]. Disruption of sleep pattern and CUD [3] might share common neurobiological pathways and mechanisms, which may help explain the improvements observed in our patients [61-64]. For example, neuroimaging in humans shows that sleep deprivation and psychostimulant intake modulate the responsiveness of dopaminergic signaling within the mesolimbic system [65-68]. Acute sleep deprivation enhances motivation for pathological reward, including drug seeking and risk taking, and might impair signaling from medial prefrontal cortex (mPFC) to nucleus accumbens (NAcc) [64]. The key role of $\mathrm{mPFC}$ in the inhibitory top-down cognitive and emotional control for the regulation of impulsivity, addictive drug seeking, and relapse has been well documented [20, 69-72]. Furthermore, a positron emission tomography (PET) study showed that impaired sleep patterns contribute to the reduction of striatal dopamine D2/D3 receptor availability in people with CUD [73]. Across species, there is a strong association between low dopamine D2 receptor levels and high impulsivity, a trait that is both a risk factor for, and a consequence of, drug abuse [74, 75]. Taken together, these findings suggest common mechanisms for sleep pattern disturbances and drug-related symptoms [76].

Another interesting observation from our study is the dose-response relationship between rTMS sessions and improvements in sleep quality, along with affect-related outcomes. Not surprisingly, our results suggest that reductions in cocaine use also accounted for these improvements, but even after we controlled for that, we found a relationship between 30-day session frequency and subsequent sleep quality. Accordingly, studies focusing on major 
depression suggest that multiple sessions of rTMS can accelerate the clinical response [56, 77].

After controlling for rTMS dosage and cocaine abstinence, we still observed a significant main effect of time (number of days since the beginning of treatment) on improvements in BDI-II, SAS, and SCL-90-R scores. The corresponding main effects of time for PSQI and CCQ scores were considerably smaller and not statistically significant. Anxiety, depressive, and negative-affect symptoms may have improved through relatively nonspecific mechanisms associated with patient entry into our clinical setting, whereas sleep disturbances and cocaine craving appeared to be more specifically responsive to rTMS dosage. Further studies are needed to investigate combined rTMS and psychotherapy for CUD. Early findings from studies on major depressive disorders show that rTMS coupled with psychotherapy leads to a considerably higher remission of clinical symptoms [78].

Finally, we observed a positive relationship between age at the first use of cocaine and current degree of anxiety (SAS score). An earlier onset of CUD has been linked to the development of cocaine addiction [79] and pattern of treatment seeking [80]. Thus, the positive relationship between the age of the first drug experience and the SAS score might be useful as a clinical measure of severity, although a deeper investigation in controlled studies is needed to comprehensively assess the role of these factors.

We are aware that the absence of a control-sham group limits the interpretation of the data, however trying to provide a better context to our results, we compared the outcomes of the recruited sample to a small group of wait-list patients who started treatment 30 days later. Results showed no significant improvement in patients of the wait-list in none of the outcome measure. Conversely, a small group of equal numerosity and with comparable clinical characteristics randomly selected from the total sample, showed a significant improvement from baseline to Day 30, in sleep disturbances, craving, anxiety, depression and other negative-affect symptoms. This is not a sham-controlled double-blind design, and thus, we cannot rule out a possible placebo effect. However, the improvement of clinical outcomes observed in our rTMS CUD cohort and the absence of any therapeutic interventions for the wait-list group suggest that rTMS, as administered here, can be considered as a therapeutic tool for CUD. Moreover, the stable clinical improvement in the 90-day period of observation is in line with our previous findings showing that rTMS treatment is accompanied by long-lasting reductions of cocaine use in a large cohort of CUD patients undergoing to the rTMS treatment and clinically followed-up for 2 years and 8 months [81].

The naturalistic clinical setting in which our cohort of CUD patients underwent the rTMS treatment provide new insights on clinical outcome measures and methodology that could be used in other drug addiction treatment clinics. However, it also carries important limitations that we acknowledge. First, as already pointed out, the absence of a control-sham group. Second, the lack of objective measures of sleep pattern changes as people with CUD may be prone to a mismatch between subjective and objective sleep quality outcomes during cocaine abstinence $[8,82]$, such that objective indices worsen while subjective quality improves. Furtger studies, including TMS- Electroencephalography (EEG) studies with sham-controlled, double-blind designs would greatly help investigate the changes in the sleep architecture of CUD subjects undergoing the rTMS treatment stimulating the left DLPFC.

\section{Conclusions}

This study provides results that are consistent with our previous findings, as well as other groups', supporting the role of rTMS, stimulating the left DLPFC, as a promising treatment of CUD, with low risk of serious adverse events [20-22, 81, 83], and disorders in comorbidity such as gambling [23]. Common self-reported withdrawal/abstinence symptoms, including sleep disturbances, anxiety, depression, and other negative affect states benefit from rTMS treatment. Future studies using standardized approach are necessary to provide more clarity on the contribution of nonspecific clinical factors. Randomized, sham-controlled clinical trials, incorporating polysomnographic recordings, are also needed to overcome the limitations of the present study and to assess working mechanisms and the prediction of treatment response.

\section{Abbreviations}

BDI-II: Beck Depression Inventory second edition; CCQ: Cocaine Craving Questionnaire; CUD: Cocaine use disorder; DLPFC: Dorsolateral prefrontal cortex; EEG: Electroencephalography; GSI: Global Severity Index; mPFC: Medial prefrontal cortex; MRI: Magnetic resonance image; NAcc: Nucleus accumbens; PET: Positron emission tomography;

PSG: Polysomnography; PSQI: Pittsburgh Sleep Quality Index; REM: Rapid eye movement sleep; rMT: Resting motor threshold; rTMS: Repetitive transcranial magnetic stimulation; SAS: Self-rating Anxiety Scale; SCL-90-R: Symptoms checklist 90-Revised; SWS: Slow-wave sleep; TMS: Transcranial magnetic stimulation

\section{Acknowledgements}

The authors would like to thank all personnel of the addiction specialty outpatient clinic from Padua (Italy) for their invaluable collaboration in the collection of data. Also, the authors would like to thank the Zardi-Gori Foundation for the fellowship bursary.

\section{Authors' contributions}

LG, AT, and LGP designed the study. GM, LG, SC, and LGP supervised and interpreted the data. GM, SC, LGP co-wrote the manuscript. SC performed the data processing and statistical analysis. TM participated in the initial data collection and analysis. AT, NC, MS, AB critically reviewed the manuscript. All authors contributed to and have approved the final manuscript.

Funding

Nothing declared. 


\section{Availability of data and materials}

The dataset used in this study are not are not publicly available due to the sensitive and personal nature of the information included. However, the corresponding author is willing to respond to any reasonable requests for de-identified data.

\section{Ethics approval and consent to participate}

Patients were informed that the data collected would be processed in accordance with the law on privacy and in compliance with Legislative Decree N. 196 of June 30, 2003, "Personal Data Protection Code", warranting anonymity. The informed consent from participants was obtained in written form. The protocol, limited to the retrospective chart review, was authorized by the Ethical Committee for the Psychological Research of the School of Psychology, University of Padova (protocol number: 2551).

\section{Consent for publication}

All participants provided written informed consent for their data to be used for research.

\section{Competing interests}

The authors declare that they have no competing interests.

\section{Author details}

${ }^{1}$ Novella Fronda Foundation, Piazza Castello, 16 -, 35141 Padua, Italy. ${ }^{2}$ Department of General Psychology, University of Padova, Padua, Italy. ${ }^{3}$ Padova Neuroscience Center, University of Padova, Padua, Italy. ${ }^{4} \mathrm{Global}$ Institutes on Addictions, Miami, FL, USA.

\section{Received: 27 October 2019 Accepted: 24 March 2020}

\section{Published online: 06 April 2020}

\section{References}

1. Grant BF, Saha TD, Ruan WJ, Goldstein RB, Chou SP, Jung J, et al. Epidemiology of DSM-5 drug use disorder: results from the National Epidemiologic Survey on alcohol and related conditions-III. JAMA psychiatry. 2016;73:39-47.

2. (EMCDDA) EMC for D and DA. European Drug Report 2019: trends and developments. 2019.

3. Angarita GA, Canavan SV, Forselius E, Bessette A, Pittman B, Morgan PT. Abstinence-related changes in sleep during treatment for cocaine dependence. Drug Alcohol Depend. 2014;134:342-7.

4. Schierenbeck T, Riemann D, Berger M, Hornyak M. Effect of illicit recreational drugs upon sleep: cocaine, ecstasy and marijuana. Sleep Med Rev. 2008;12:381-9.

5. Mahoney JJ, De La Garza R, Jackson BJ, Verrico CD, Ho A, lqbal T, et al. The relationship between sleep and drug use characteristics in participants with cocaine or methamphetamine use disorders. Psychiatry Res. 2014;219:367-71.

6. Hodges SE, Pittman B, Morgan PT. Sleep perception and misperception in chronic cocaine users during abstinence. Sleep. 2017;40. https://doi.org/10. 1093/sleep/zsw069.

7. Johanson CE, Roehrs T, Schuh K, Warbasse L. The effects of cocaine on mood and sleep in cocaine-dependent males. Exp Clin Psychopharmacol. 1999;7:338-46

8. Morgan PT, Pace-Schott EF, Sahul ZH, Coric V, Stickgold R, Malison RT. Sleep, sleep-dependent procedural learning and vigilance in chronic cocaine users: evidence for occult insomnia. Drug Alcohol Depend. 2006;82:238-49.

9. Thompson PM, Gillin JC, Golshan S, Irwin M. Polygraphic sleep measures differentiate alcoholics and stimulant abusers during short-term abstinence. Biol Psychiatry. 1995;38:831-6.

10. Angarita GA, Canavan SV, Forselius E, Bessette A, Morgan PT. Correlates of polysomnographic sleep changes in cocaine dependence: self-administration and clinical outcomes. Drug Alcohol Depend. 2014;143:173-80.

11. Berro LF, Frussa-Filho R, Tufik S, Andersen ML. Relationships between sleep and addiction: the role of drug-environment conditioning. Med Hypotheses. 2014;82:374-6.

12. Demontis MG, Fadda P, Devoto P, Martellotta MC, Fratta W. Sleep deprivation increases dopamine D1 receptor antagonist [3H] SCH 23390 binding and dopamine-stimulated adenylate cyclase in the rat limbic system. Neurosci Lett. 1990;117:224-7.

13. Fadda P, Martellotta MC, Gessa GL, Fratta W. Dopamine and opioids interactions in sleep deprivation. Prog Neuro-Psychopharmacol Biol Psychiatry. 1993;17:269-78.
14. Nunes Júnior GP, Tufik S, Nobrega JN. Autoradiographic analysis of D1 and D2 dopaminergic receptors in rat brain after paradoxical sleep deprivation. Brain Res Bull. 1994;34:453-6.

15. Gujar N, Yoo S-S, Hu P, Walker MP. Sleep deprivation amplifies reactivity of brain reward networks, biasing the appraisal of positive emotional experiences. J Neurosci. 2011:31:4466-74.

16. Koob GF, Volkow ND. Neurobiology of addiction: a neurocircuitry analysis. Lancet Psychiatry. 2016;3:760-73.

17. Barker AT, Jalinous R, Freeston IL. Non-invasive magnetic stimulation of human motor cortex. Lancet. 1985:325:1106-7.

18. Leuchter AF, Cook IA, Jin Y, Phillips B. The relationship between brain oscillatory activity and therapeutic effectiveness of transcranial magnetic stimulation in the treatment of major depressive disorder. Front Hum Neurosci. 2013;7:37.

19. Diana M, Raij T, Melis M, Nummenmaa A, Leggio L, Bonci A. Rehabilitating the addicted brain with transcranial magnetic stimulation. Nat Rev Neurosci. 2017;18:685-93.

20. Terraneo A, Leggio L, Saladini M, Ermani M, Bonci A, Gallimberti L. Transcranial magnetic stimulation of dorsolateral prefrontal cortex reduces cocaine use: a pilot study. Eur Neuropsychopharmacol. 2016;26:37-44.

21. Rapinesi C, Del Casale A, Di Pietro S, Ferri VR, Piacentino D, Sani G, et al. Add-on high frequency deep transcranial magnetic stimulation (dTMS) to bilateral prefrontal cortex reduces cocaine craving in patients with cocaine use disorder. Neurosci Lett. 2016;629:43-7.

22. Politi E, Fauci E, Santoro A, Smeraldi E. Daily sessions of transcranial magnetic stimulation to the left prefrontal cortex gradually reduce cocaine craving. Am J Addict. 2008;17:345-6.

23. Cardullo S, Gomez Perez JL, Marconi L, Terraneo A, Gallimberti L, Bonci A, et al. Clinical improvements in comorbid gambling/cocaine use disorder (GD/CUD) patients undergoing repetitive Transcranial magnetic stimulation (rTMS). J Clin Med. 2019;8:768.

24. Bolloni C, Panella R, Pedetti M, Frascella AG, Gambelunghe C, Piccoli T, et al. Bilateral transcranial magnetic stimulation of the prefrontal cortex reduces cocaine intake: a pilot study. Front Psychiatry. 2016;7:133

25. Cohrs S, Tergau F, Riech S, Kastner S, Paulus W, Ziemann U, et al. Highfrequency repetitive transcranial magnetic stimulation delays rapid eye movement sleep. Neuroreport. 1998;9:3439-43.

26. Huber R, Esser SK, Ferrarelli F, Massimini M, Peterson MJ, Tononi G. TMSinduced cortical potentiation during wakefulness locally increases slow wave activity during sleep. PLoS One. 2007;2:e276.

27. Nardone R, Höller Y, Brigo F, Tezzon F, Golaszewski S, Trinka E. Transcranial magnetic stimulation and sleep disorders: pathophysiologic insights. Sleep Med. 2013;14:1047-58. https://doi.org/10.1016/j.sleep.2013.04.025.

28. Gang JC, Zhang T, Guo YF, Ling YM, Gao D. Efficacy of repetitive Transcranial magnetic stimulation in the treatment of patients with chronic primary insomnia. Cell Biochem Biophys. 2013;67:169-73.

29. Lin J, Liu X, Li H, Yu L, Shen M, Lou Y, et al. Chronic repetitive transcranial magnetic stimulation (rTMS) on sleeping quality and mood status in drug dependent male inpatients during abstinence. Sleep Med. 2019;58:7-12.

30. Pettorruso M, Martinotti G, Santacroce R, Montemitro C, Fanella F, Di Giannantonio M. rTMS reduces psychopathological burden and cocaine consumption in treatment-seeking subjects with cocaine use disorder: an open label, feasibility study. Front psychiatry. 2019;10:621.

31. American Psychiatric Association. Diagnostic and Statistical Manual of Mental Disorders: DSM-5; 2013. https://doi.org/10.1176/appi.books. 9780890425596.744053.

32. Buysse DJ, Reynolds CF, Monk TH, Berman SR, Kupfer DJ. The Pittsburgh sleep quality index: a new instrument for psychiatric practice and research. Psychiatry Res. 1989;28:193-213. https://doi.org/10.1016/0165-1781(89)90047-4.

33. Mollayeva T, Thurairajah P, Burton K, Mollayeva S, Shapiro CM, Colantonio A. The Pittsburgh sleep quality index as a screening tool for sleep dysfunction in clinical and non-clinical samples: a systematic review and meta-analysis. Sleep Med Rev. 2016;25:52-73.

34. Weiss RD, Griffin ML, Hufford C, Muenz LR, Najavits LM, Jansson SB, et al. Early prediction of initiation of abstinence from cocaine. Am J Addict. 1997; 6:224-31. https://doi.org/10.1111/j.1521-0391.1997.tb00401.X.

35. Beck A, Steer R, Brown G. Beck depression inventory-II. San Antonio: Psychological Corporation; 1996. http://www.pearsonclinical.com/ psychology/products/100000159/beck-depression-inventoryii-bdi-ii.html? Pid $=015-8018-370$.

36. Zung WWK A rating instrument for anxiety disorders. Psychosomatics. 1971;12:371-9.

37. Derogatis LR. Symptom Checklist-90-R (SCL-90-R): administration, scoring, and procedures manual. 3rd ed. Minneapolis: MN NCS Pearson; 1994. 
38. RStudio Team. RStudio: integrated development for R. Boston, MA: RStudio Inc; 2016.

39. R Core Team. R. A language and environment for statistical computing. Vienna: R Found Stat Comput; 2018.

40. Bates $D$, Mächler M, Bolker B, Walker S. Fitting linear mixed-effects models using Ime4. J Stat Softw. 2015.

41. Kuznetsova A, Brockhoff PB, Christensen RHB. ImerTest package: Tests in linear mixed effects models. J Stat Softw. 2017;82:13.

42. Fox J. Effect displays in R for generalised linear models. J Stat Softw. 2003:8:1-27.

43. Rosnow RL, Rosenthal R, Rubin DB. Contrasts and correlations in effect-size estimation. Psychol Sci. 2000;11:446-53. https:/doi.org/10.1111/1467-9280.00287.

44. Brower KJ, Perron BE. Sleep disturbance as a universal risk factor for relapse in addictions to psychoactive substances. Med Hypotheses. 2010;74:928-33.

45. Angarita GA, Emadi N, Hodges S, Morgan PT. Sleep abnormalities associated with alcohol, cannabis, cocaine, and opiate use: a comprehensive review. Addict Sci Clin Pract. 2016;11:9.

46. Brower KJ, Maddahian E, Blow FC, Beresford TP. A comparison of selfreported symptoms and DSM-III-R criteria for cocaine withdrawal. Am J Drug Alcohol Abuse. 1988;14:347-56.

47. Morgan PT, Angarita GA, Canavan S, Pittman B, Oberleitner L, Malison RT, et al. Modafinil and sleep architecture in an inpatient-outpatient treatment study of cocaine dependence. Drug Alcohol Depend. 2016;160:49-56.

48. Dackis CA, Kampman KM, Lynch KG, Pettinati HM, O'brien CP. A doubleblind, placebo-controlled trial of modafinil for cocaine dependence. Neuropsychopharmacology. 2005;30:205-11.

49. Kampman KM, Lynch KG, Pettinati HM, Spratt K, Wierzbicki MR, Dackis C, et al. A double blind, placebo controlled trial of modafinil for the treatment of cocaine dependence without co-morbid alcohol dependence. Drug Alcohol Depend. 2015;155:105-10.

50. Anderson AL, Reid MS, Li S-H, Holmes T, Shemanski L, Slee A, et al. Modafinil for the treatment of cocaine dependence. Drug Alcohol Depend. 2009;104:133-9.

51. Dackis CA, Kampman KM, Lynch KG, Plebani JG, Pettinati HM, Sparkman T, et al. A double-blind, placebo-controlled trial of modafinil for cocaine dependence. J Subst Abus Treat. 2012;43:303-12.

52. Schmitz JM, Rathnayaka N, Green C, Moeller FG, Dougherty AE, Grabowski J. Combination of modafinil and d-amphetamine for the treatment of cocaine dependence: a preliminary investigation. Front psychiatry. 2012;3:77.

53. Schmitz JM, Green CE, Stotts AL, Lindsay JA, Rathnayaka NS, Grabowski J, et al. A two-phased screening paradigm for evaluating candidate medications for cocaine cessation or relapse prevention: Modafinil, levodopa-carbidopa, naltrexone. Drug Alcohol Depend. 2014;136:100-7.

54. Liang Y, Wang L, Yuan T-FF. Targeting withdrawal symptoms in men addicted to methamphetamine with Transcranial magnetic stimulation: a randomized clinical trial. JAMA Psychiatry. 2018;75:1199-201.

55. Su H, Zhong N, Gan H, Wang J, Han H, Chen T, et al. High frequency repetitive transcranial magnetic stimulation of the left dorsolateral prefrontal cortex for methamphetamine use disorders: a randomised clinical trial. Drug Alcohol Depend. 2017;175:84-91.

56. Gershon AA, Dannon PN, Grunhaus L. Transcranial magnetic stimulation in the treatment of depression. Am J Psychiatry. 2003;160:835-45.

57. Donse L, Sack AT, Fitzgerald PB, Arns M. Sleep disturbances in obsessivecompulsive disorder: association with non-response to repetitive transcranial magnetic stimulation (rTMS). J Anxiety Disord. 2017:49:31-9. https://doi.org/10.1016/j.janxdis.2017.03.006

58. Sánchez-Escandón O, Arana-Lechuga Y, Terán-Pérez G, Ruiz-Chow A, GonzálezRobles $R$, Shkurovich-Bialik $P$, et al. Effect of low-frequency repetitive transcranial magnetic stimulation on sleep pattern and quality of life in patients with focal epilepsy. Sleep Med. 2016;20:37-40. https:/doi.org/10.1016/j.sleep.2015.11.022.

59. Pellicciari MC, Cordone S, Marzano C, Bignotti S, Gazzoli A, Miniussi C, et al. Dorsolateral prefrontal transcranial magnetic stimulation in patients with major depression locally affects alpha power of REM sleep. Front Hum Neurosci. 2013;7:433. https://doi.org/10.3389/fnhum.2013.00433.

60. Zhang C, Liu J. Effects of Transcranial magnetic stimulation on Polysomnography in patients with insomnia. Chinese J Rehabil Theory Pract 2013;0:568-571.

61. Oishi Y, Lazarus M. The control of sleep and wakefulness by mesolimbic dopamine systems. Neurosci Res. 2017;118:66-73. https://doi.org/10.1016/j.neures.2017.04.008.

62. Eban-Rothschild A, Rothschild G, Giardino WJ, Jones JR, De Lecea L. VTA dopaminergic neurons regulate ethologically relevant sleep-wake behaviors. Nat Neurosci. 2016;19:1356-66. https://doi.org/10.1038/nn.4377.

63. Pereira AC, Mao X, Jiang CS, Kang G, Milrad S, McEwen BS, et al. Dorsolateral prefrontal cortex GABA deficit in older adults with sleep-disordered breathing. Proc Natl Acad Sci. 2017;114:10250-5. https//doi.org/10.1073/pnas.1700177114.
64. Liu Z, Wang Y, Cai L, Li Y, Chen B, Dong Y, et al. Prefrontal cortex to Accumbens projections in sleep regulation of reward. J Neurosci. 2016;36: 7897-910. https://doi.org/10.1523/JNEUROSCI.0347-16.2016.

65. Berro LF, Santos R, Hollais AW, Wuo-Silva R, Fukushiro DF, Mári-Kawamoto E, et al. Acute total sleep deprivation potentiates cocaine-induced hyperlocomotion in mice. Neurosci Lett. 2014;579:130-3.

66. Drevets WC, Gautier C, Price JC, Kupfer DJ, Kinahan PE, Grace AA, et al. Amphetamine-induced dopamine release in human ventral striatum correlates with euphoria. Biol Psychiatry. 2001;49:81-96.

67. Volkow ND, Wang G, Telang F, Fowler J, Logan J, Wong C, et al. Sleep deprivation decreases binding of $[11 \mathrm{C}]$ raclopride to dopamine D2/D3 receptors in the human brain. J Neurosci. 2008;28:8454-61.

68. Volkow ND, Wang GJ, Fowler JS, Gatley SJ, Ding YS, Logan J, et al Relationship between psychostimulant-induced "high" and dopamine transporter occupancy. Proc Natl Acad Sci. 1996;93:10388-92.

69. Chen BT, Yau HJ, Hatch C, Kusumoto-Yoshida I, Cho SL, Hopf FW, et al. Rescuing cocaine-induced prefrontal cortex hypoactivity prevents compulsive cocaine seeking. Nature. 2013;496:359-62.

70. Goldstein RZ, Volkow ND. Drug addiction and its underlying neurobiological basis: neuroimaging evidence for the involvement of the frontal cortex. Am J Psychiatry. 2002;159:1642-52.

71. Castelluccio BC, Meda SA, Muska CE, Stevens MC, Pearlson GD. Error processing in current and former cocaine users. Brain Imaging Behav. 2014;8:87-96.

72. Tononi G, Cirelli C. Sleep and the Price of plasticity: from synaptic and cellular homeostasis to memory consolidation and integration. Neuron. 2014;81:12-34. https://doi.org/10.1016/j.neuron.2013.12.025.

73. Wiers CE, Shumay E, Cabrera E, Shokri-Kojori E, Gladwin TE, Skarda E, et al. Reduced sleep duration mediates decreases in striatal D2/D3 receptor availability in cocaine abusers. Transl Psychiatry. 2017;6:e752.

74. Everitt BJ. Neural and psychological mechanisms underlying compulsive drug seeking habits and drug memories - indications for novel treatments of addiction. Eur J Neurosci. 2014;40:2163-82.

75. Jentsch JD, Ashenhurst JR, Cervantes MC, Groman SM, James AS, Pennington ZT. Dissecting impulsivity and its relationships to drug addictions. Ann N Y Acad Sci. 2014;1327:1-26.

76. Strafella AP, Paus T, Barrett J, Dagher A. Repetitive transcranial magnetic stimulation of the human prefrontal cortex induces dopamine release in the caudate nucleus. J Neurosci. 2001;21:1-4.

77. Modirrousta M, Meek BP, Wikstrom SL. Efficacy of twice-daily vs once-daily sessions of repetitive transcranial magnetic stimulation in the treatment of major depressive disorder: a retrospective study. Neuropsychiatr Dis Treat. 2018;14:309-16. https://doi.org/10.2147/NDT.S151841.

78. Donse L, Padberg F, Sack AT, Rush AJ, Arns M. Simultaneous rTMS and psychotherapy in major depressive disorder: clinical outcomes and predictors from a large naturalistic study. Brain Stimul. 2018;11:337-45.

79. Wagner FA, Anthony JC. From first drug use to drug dependence: developmental periods of risk for dependence upon marijuana, cocaine, and alcohol. Neuropsychopharmacology. 2002;26:479-88.

80. Kessler RC, Aguilar-Gaxiola S, Berglund PA, Caraveo-Anduaga JJ, DeWit DJ, Greenfield SF, et al. Patterns and predictors of treatment seeking after onset of a substance use disorder. Arch Gen Psychiatry. 2001;58:1065-71.

81. Madeo G, Terraneo A, Cardullo S, Gómez Pérez L, Cellini N, Sarlo M, et al. Long-term outcome of repetitive transcranial magnetic stimulation in a large cohort of patients with cocaine-use disorder: an observational study. Front Psychiatry. 2020;11:158

82. Pace-Schott EF, Stickgold R, Muzur A, Wigren PE, Ward AS, Hart CL, et al. Sleep quality deteriorates over a binge-abstinence cycle in chronic smoked cocaine users. Psychopharmacology (Berl). 2005;179:873-83.

83. Pettorruso M, Spagnolo PA, Leggio L, Janiri L, Di Giannantonio M, Gallimberti L, et al. Repetitive transcranial magnetic stimulation of the left dorsolateral prefrontal cortex may improve symptoms of anhedonia in individuals with cocaine use disorder: a pilot study. Brain Stimul. 2018;11:1195-7.

\section{Publisher's Note}

Springer Nature remains neutral with regard to jurisdictional claims in published maps and institutional affiliations. 